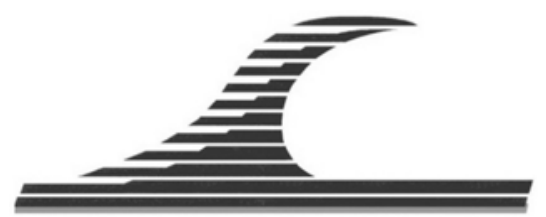

Revue Paralia, Volume 8 (2015) pp s01.1-s01.7

Keywords: Coastal and port infrastructure, Maritime works, Quays, Ground anchors, Non destructive testing, Tensile force,

Foundations, Sheet piles, Maintenance. (C) Editions Paralia CFL

\title{
Innovative non-destructive technique for determining tension in ground anchors
}

\author{
Laurent MITAINE ${ }^{1}$, Jean-Jacques RINCENT ${ }^{2}$
}

1. Rincent Ports, 39 rue Michel-Ange 91026 Evry Cedex, France. laurent.mitaine@rincentports.com

2. Rincent BTP Services SAS, 39 rue Michel-Ange 91026 Evry Cedex, France. jean-jacques.rincent@rincent.com

\begin{abstract}
:
In order to facilitate long-term maintenance of coastal and port infrastructures, this technique allows measuring and determining tension in ground anchors that support maritime and port retaining walls such as sheet piles or diaphragm walls.

It applies both for final acceptance of works and for monitoring the residual tension in tendons during the life of the structure.

This technique has three benefits:

- it is non-destructive, as opposed to usual extra-loading technique that destroys or damage $10 \%$ of the ground anchors. It uses patented mechanical impedance instrumentation, which is manipulated by only one operator. It does not need heavy equipment;

- it allows testing an important quantity of ground anchors in shorter time, when usual extra-loading technique needs more logistics and forces to work only through anchor sampling;

- it works even under water, requiring only one diver.

This technique then allows testing and checking all the ground anchors of a quay (or any other type of infrastructure using ground anchors), much faster than usual extraloading technique and with excellent safety conditions.
\end{abstract}

Translated version of a paper in French (DOI reference: http://dx.doi.org/10.5150/cmcm.2015.052), presented in the Coastal and Maritime Mediterranean Conference, edition 3, 25-27 November 2015, Ferrara, Italy. Received 04 September 2015, accepted 11 December 2015, available online 28 December 2015.

How to cite this paper:

MITAINE L., RINCENT J.-J. (2015). Innovative non-destructive technique for determining tension in ground anchors. Revue Paralia, Vol. 8, pp s01.1-s01.7.

DOI: http://dx.doi.org/10.5150/revue-paralia.2015.s01 


\section{Introduction - Operating principle of this non-destructive method}

This non-destructive technique of determining the effective tension in ground anchors can be used both for acceptance of works, or for monitoring residual tension in the case of maintenance diagnosis. It is made possible by the analysis of the vibrational response of an element set into vibration by means of an impact whose strength is measured. This element is assimilated as an elastic spring set into vibration (DAVIS et al., 1974). The obtained vibrational response is function of:

- the shape, inertia, drilling diameter, tendon length, nature of its constituting materials;

- surrounding materials, such as metal sheet piling, reinforced concrete diaphragm wall...;

- the tendon tensile force.

\section{Test implementation and equipment required}

The equipment required for this testing is schematically shown in Figure 1:

- an impactor that is equipped with a strength sensor, which hits in tendon's axis;

- a velocity or acceleration sensor (such as a magnet-on-spring geophone) which is positioned on the bearing plate or else on the anchor head;

- a mathematical treatment of the acquisitions to get the frequency curve (see Figure 2).

The testing implementation consists to:

1) access the operating area;

2) set the operator that will control the acquisitions from a computer (laptop);

3) brush and clean the bearing plate, prior to testing, to allow both adequate connection of the sensor and impactor strike on a clean surface;

4) fix the sensor with a coupling product on the bearing plate of the ground anchor. The coupling product used can be universal lithium grease, whose use is validated by the competent authorities in terms of hygiene and health at work. The sensor is also adapted to inclined tendons (meaning a non-symmetrical response);

5) set into vibration the ground anchor with a strike induced on its bearing plate, in its axis' direction. For each tested ground anchor, a minimum of 6 acquisitions is made, each being the combination of two tests, in order to reduce parasitic vibrations and to amplify the vibrational response of the tendon.

6) record $F$ impact force,

7) record V vibration of the system ("system" being the ground anchor and the sheet pile or the diaphragm wall) through the sensor which is linked to the bearing plate.

Complementary measurements are implemented for the determination of the tensile stress in the tendon, which requires the completion of further non-destructive testing from the intermediate concrete hump between the sheet pile (or the diaphragm wall) and the bearing plate at the head of the stressed tendon, in order to identify the dynamic stiffness linked to the rigidity of this concrete block. 
The equipment and the procedure for this test are the same as for testing on the bearing plates.

Similarly, additional tests are performed on the sheet pile or on the diaphragm wall, in order to isolate their part of dynamic stiffness.

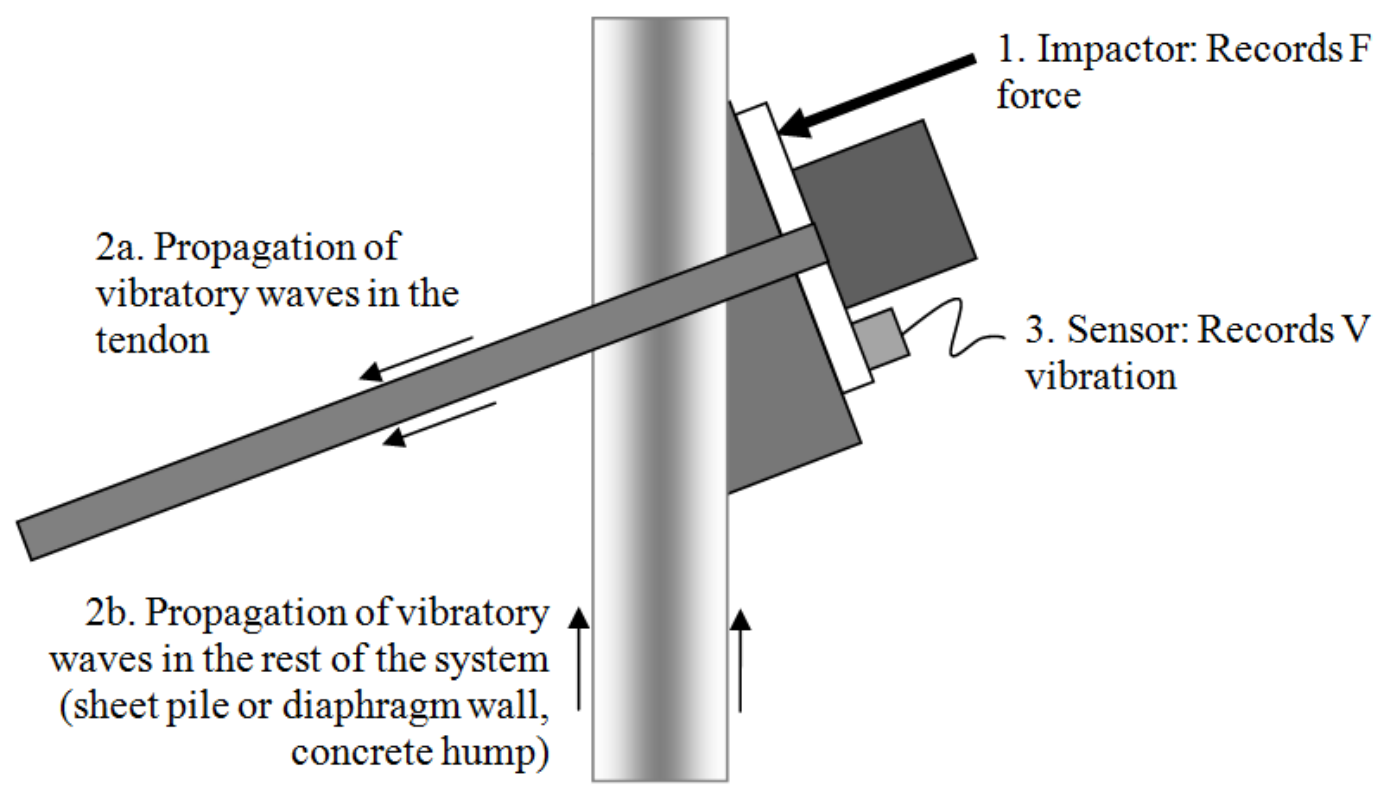

Figure 1. Testing implementation of a ground anchor.

\section{Determination of anchor lengths by analyzing its vibratory response}

Vibratory response analysis focuses on "frequency response" and "stiffness" parameters of the frequency curve obtained following the acquisition (see typical curve in Figure 2).

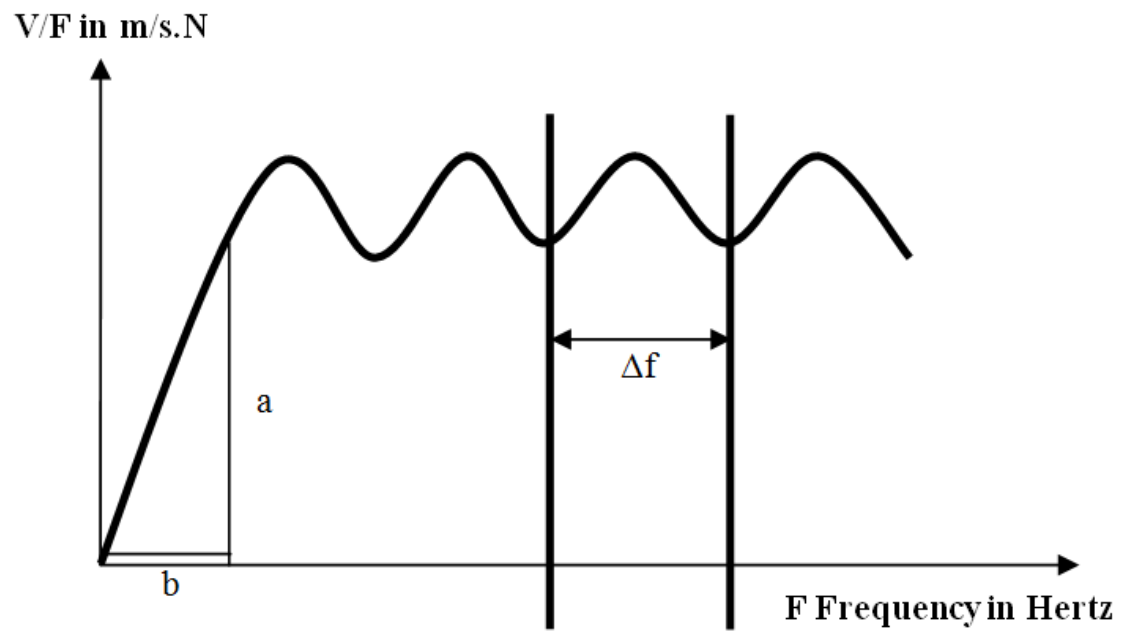

Figure 2. Typical curve after acquisition treatment. 
The curve of successive maximal and minimum amplitudes is positioned in frequency following the system natural vibrating frequency, according to the principle of superposition of sinusoidal curves.

Decomposition of the curves:

Fourier's theorem defines that any $f_{n}$ periodic function of a $\tau$ period can be written according to equation 1 :

$z(t)=\sum_{n} A_{n} \sin \left(2 \pi f_{n}+\phi_{n}\right)$

where: $f_{n}=n f_{l}$ and $f_{l}=1 / \tau$

Under these conditions a curve is obtained where a Speed-Force data varies as function of a frequency.

The length of the tested element is obtained by equation 2 :

$L=\frac{V}{2 \Delta F}$

where: $L$ is a length, $V$ is the speed of the waves propagation in the anchor and $\Delta F$ is the frequency spread between two successive maximal or minimal amplitudes.

It can be one or several dominant vibrational regimes, which eventually allow the determination of both the total length of the tendon and its unbonded length, according to Figure 3.

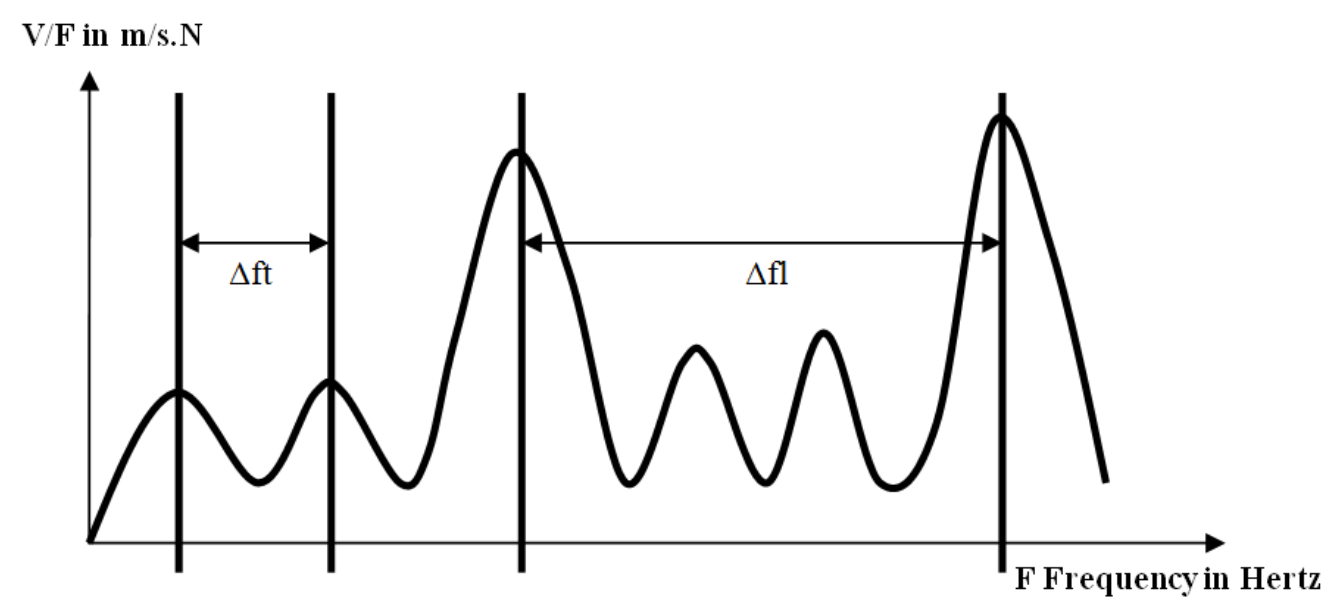

Figure 3. Lengths analysis from curve

The vibration of the tendon unbonded length induces a $\Delta F_{l}$ period on the curve, which is transformed into a $\mathrm{L}_{1}$ unbonded length given by Eq. 3:

$L_{l}=\frac{V}{2 \Delta F_{l}}$

In addition, the vibration of the tendon whole length induces a $\Delta F_{t}$ period of successive peaks on the curve, which is transformed into a $L_{t}$ total tendon length given by Eq. 4 : 


$$
L_{t}=\frac{V}{2 \Delta F_{t}}
$$

\section{Determination of the tendon stiffness through frequency response analysis}

The dynamic stiffness (given in $\mathrm{N} / \mathrm{m}$ ) which is proportional to the inverse of the slope of the curve at its origin ( $2 \pi b / a$ when taking the slope values $a$ and $b$ from Figure 2) depends on the tested system inertia and its direct environment.

Dynamic stiffness is a complex number with a real part and an imaginary part that can be represented as a sum-vector composed of a stiffness in the orthogonal direction of the application of the load and of a stiffness in the direction of the applied load.

Static stiffness in itself is the value of the slope at a point on the force/deformation curve, i.e. under a given tensile force. Its unit is $\mathrm{N} / \mathrm{m}$ and it is the value that we are looking for.

The dynamic stiffness of an element increases with the tension force which is applied thereto, that eventually implies a reduction of the amplitude of its vibration frequency curve, according to Figure 4 (note that the tensile force values indicated for each curve are given as an example).

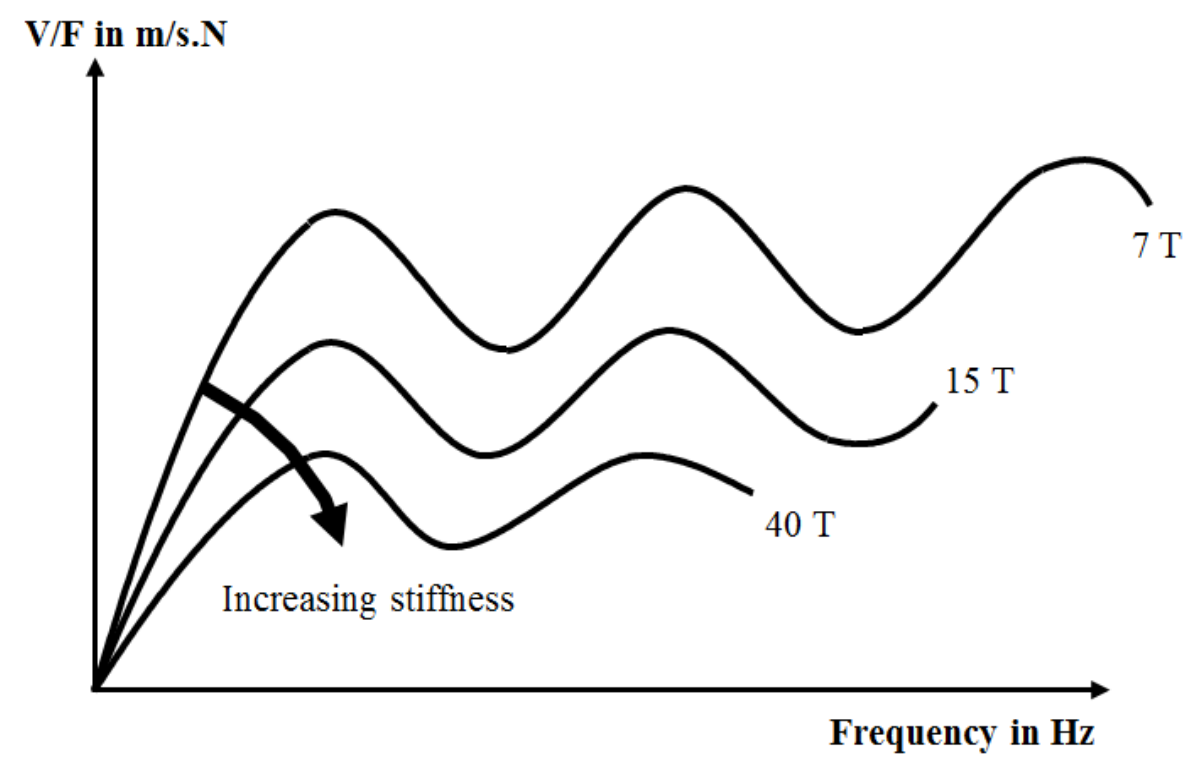

Figure 4. Reduction of the amplitude of the vibrational frequency curves of an object related to the increase in tensile force applied thereto

In the case of an anchor tendon, it depends on its type and its geometrical characteristics, on its anchor grout length and characteristics, and on tensile forces applied.

A "correct" stiffness value shows:

- a proper bonding of the anchor tendon; 
- the absence of a failure;

- the continuity of the anchor along its total length.

Low stiffness highlights disorders:

- at the head of the anchor, meaning a potential failure or loss of tension;

- a failure at bonding's level or in the tendon.

Field experience gained in this type of diagnosis shows that there is about a 10 times ratio between "correct" stiffness and "weak" stiffness.

This parameter is correlated to the static stiffness, that is to say, the slope at a point on the curve force/deformation after a direct tension load test.

Dynamic stiffness is then directly related to the tensile force in the anchor. The comparison between the stiffness which is obtained in tested anchors and the dynamic stiffness/tensile force relations from Rincent BTP Services database allows estimating the real tensile force in the anchor tendon with a less than $5 \%$ error.

\section{Conclusion}

At the end of data analysis, output data are:

- tendon's unbonded and total lengths, and their average calculated from acquisitions;

- measured stiffness and its average calculated from acquisitions;

- stiffness interpretation for determining tensile force applied to the tendon.

This type of test and the associated patent (RINCENT BTP SERVICES, 2006) applies to tendons, strands, bars, nails and more generally to all types of anchored reinforcements. Besides its usefulness for civil infrastructures, it is also applicable to the verification of machine-tool installation anchorage in industries.

In addition, it should be noted that this type of non-destructive testing is suitable for in situ measurement of fatigue phenomena, since it determines the tensile fluctuations after the intake of cyclic stresses, without destroying the tested item.

In conclusion, this type of non-destructive testing allows to determine precisely and efficiently the most representative characteristics of all types of ground anchor, particularly in the context of maritime and port infrastructure, and can be used as an instrumented technique for:

- final acceptance of anchors works, giving the possibility of a comprehensive and detailed control of all tendons,

- monitoring of the residual tension in tendons during the life and maintenance of quays and other retaining structures, allowing a regular periodicity at a reduced cost of measuring under-tension or over-tension in existing tendons, and then preventing the risks of failure or collapse of structures.

Its speed of implementation allows exhaustive measurement of all a series of anchors, where traditional solutions -which require more logistics- work only by sampling. 


\section{References}

RINCENT BTP SERVICES (2006). Method and device for determining the tensile stress exerted on a sealed element. Patent OMPI n ${ }^{\circ}$ WO 2006/010830 A1, 2 February 2006.

DAVIS A.G., DUNN C.S., CEBTP (1974). From theory to field experience with the non-destructive vibration testing of piles. Proceedings of the Institution of Civil Engineers, Part 2, Vol. 57(4), pp 571-593. http://dx.doi.org/10.1680/iicep.1974.3895 